\title{
Structure of Small World Innovation Network and Learning Performance
}

\author{
Shuang Song, ${ }^{1,2}$ Xiangdong Chen, ${ }^{1}$ and Gupeng Zhang $^{3}$ \\ ${ }^{1}$ School of Economics and Management, Beihang University, Beijing 100191, China \\ ${ }^{2}$ Library, Beihang University, Beijing 100191, China \\ ${ }^{3}$ College of Technology Management, University of Chinese Academy of Science, Beijing 100049, China
}

Correspondence should be addressed to Gupeng Zhang; zhanggupeng@163.com

Received 9 April 2014; Accepted 13 May 2014; Published 2 June 2014

Academic Editor: L. W. Zhang

Copyright (C) 2014 Shuang Song et al. This is an open access article distributed under the Creative Commons Attribution License, which permits unrestricted use, distribution, and reproduction in any medium, provided the original work is properly cited.

\begin{abstract}
This paper examines the differences of learning performance of $5 \mathrm{MNCs}$ (multinational corporations) that filed the largest number of patents in China. We establish the innovation network with the patent coauthorship data by these 5 MNCs and classify the networks by the tail of distribution curve of connections. To make a comparison of the learning performance of these $5 \mathrm{MNCs}$ with differing network structures, we develop an organization learning model by regarding the reality as having $m$ dimensions, which denotes the heterogeneous knowledge about the reality. We further set $n$ innovative individuals that are mutually interactive and own unique knowledge about the reality. A longer (shorter) distance between the knowledge of the individual and the reality denotes a lower (higher) knowledge level of that individual. Individuals interact with and learn from each other within the smallworld network. By making 1,000 numerical simulations and averaging the simulated results, we find that the differing structure of the small-world network leads to the differences of learning performance between these 5 MNCs. The network monopolization negatively impacts and network connectivity positively impacts learning performance. Policy implications in the conclusion section suggest that to improve firm learning performance, it is necessary to establish a flat and connective network.
\end{abstract}

\section{Introduction}

Many studies have proved that the innovation process has recently become a network process [1]. Innovation network facilitates knowledge sharing, R\&D cooperation, and technology complementation between firms, while constraints like distance and culture are playing less important roles. The contacts of firms from different regions and countries are becoming closer, which characterizes the "small world" of innovation network. As the theory of "six-degrees-ofseparation" (everyone is six or fewer steps away, by way of introduction, from any other person in the world, so that a chain of "a friend of a friend" statements can be made to connect any two people in a maximum of six steps) proposed by Milgram [2] has been becoming more popular, the closely related theory of "small world" has been receiving much focus [3]. In the small world network, most vertices are far away from each other but could reach each other by passing several other vertices. If we denote the vertices in the small world network by people, edges between vertices suggest that the two people know each other; then the network where everyone could know each other by a chain of "a friend of a friend" is signified by the phenomenon of "small world."

The social network is so complex and important that a comprehensive study from the fields of computer science, physics, statistics, sociology, and even economics is carried out [4]. The literature on small worlds has grown rapidly in the social science and management literature [5], like Björneborn [6], Davis et al. [7], Fowler [8], Kogut and Walker [9], Kleinberg [10], Iravani et al. [11], Goyal et al. [12], Baum et al. [13], and Watts [14]. In recent years, many scholars introduced small world theory into innovation network and focused on the "small worldliness" of innovation network, for example, Fleming and Marx [15], Hargadon [16], Chen and Guan [17], Guan and Shi [18], Fleming et al. [19], and Hung and Wang [20]. In a regular innovation network, individuals are constrained by distance and thus can only connect with close neighbors; see Figure 1(a). In small world 


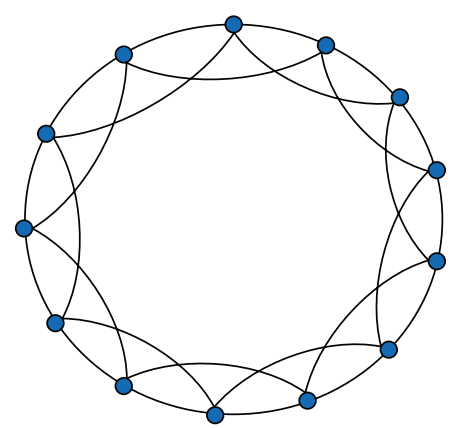

(a) Regular

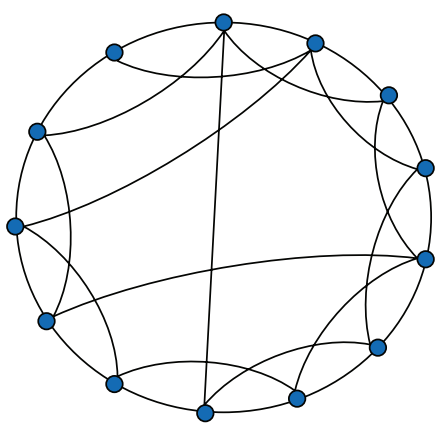

(b) Small world

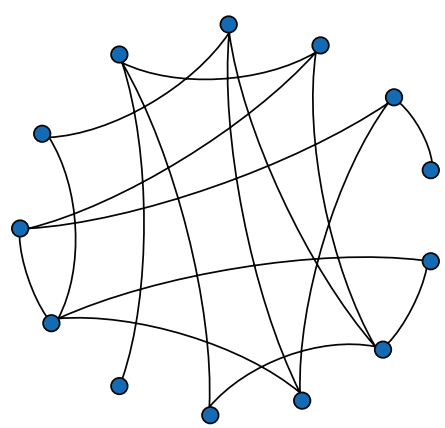

(c) Random

FIGURE 1: Three types of innovation network.

innovation network, minor connections are established by several individuals far from each other; see Figure 1(b). In random innovation network, the connections are totally random and free of distance; see Figure 1(c). Obviously, small world innovation network lies between regular and random innovation networks; that is, most connections are constrained by but some are free of distance. As most real innovation networks lie between regular and random networks, it is common to see small world innovation network, which is playing an important role in the technology progress of firms and even the whole industry $[15,21]$.

The network system is important to an organization [22, 23]. The structure of small-world networks and of real networks has been probed through the calculation of their diameter as a function of network size [24]. Based on the analysis of the small world network, electric power grid for Southern California, the network of movie-actor collaborations, the neuronal network of the worm Caenorhabditiselegans [25], the world-wide web [26], and the network of citations of scientific papers $[27,28]$, the scale-free network with the distribution of connectivities that decays with a power law tail is proposed. Scale-free networks are also small-world networks, because (i) they have clustering coefficients much larger than random networks [24] and (ii) their path length increases logarithmically with the number of vertices [26]. However, as there are constraints limiting the addition of new links, Amaral et al. [29] suggested that such constraints may be the controlling factor (e.g., aging of vertices and cost of adding new links) for the emergence of scale-free networks; they further presented evidence of the occurrence of three classes of small-world networks according to the constraints: (a) scale-free networks with no constraints, characterized by a vertex connectivity distribution that decays as a power law; (b) broad-scale networks with low constraints, characterized by a connectivity distribution that has a power law regime followed by a sharp cutoff; and (c) single-scale networks with high constraints, characterized by a connectivity distribution with a fast decaying tail (see Figure 2).

It is increasingly acknowledged that network structure plays an important role in explaining the potential of emerging technologies to spread [30-32]. Many studies focused on the patent coauthorship network and the innovation productivity of inventors, for example, Chen and Guan [17],

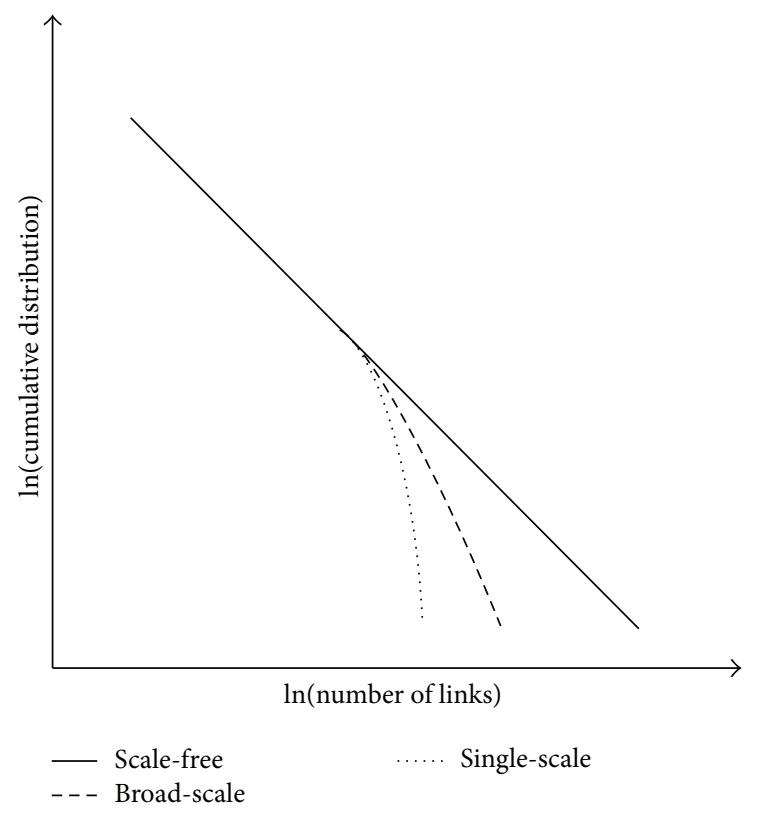

Figure 2: Truncation of scale-free connectivity by adding constraints to network.

Fleming et al. [19], and Zhang and Guan [33]. As the patents are mostly coinvented by inventors with complementary knowledge, inventors could gain the necessary knowledge by making a joint research, where the network relationships between inventors are functioning as the intermediaries of knowledge flow. Patent coauthorship network, which reflects the networking relationship between inventors, plays an important role in facilitating the knowledge flow. Inventors are continuously learning from each other through the networks and improve their own knowledge, which in turn improves the organization learning [34].

Networks with different structures would play different roles in improving learning performance [35]. Numerous studies on the network effects between diverse firms have been conducted, focusing on the way in which innovation clusters are continuously achieving competitiveness and improving organization learning performance [36-41]. Finding the effective structure of innovation networks within 
the firm may be indicative for firm managers who care about the organization design.

Existing studies may not make an in-depth analysis of the differences of the network structure between firms, leaving an unclear effectiveness of the networks with differing structures. However, measuring and comparing the competitiveness of innovation networks with the real regional firm data may be difficult, since there are other potential multiimpacting factors, for example, R\&D input, economic profit, wages, and culture, which make it difficult to identify the effect of innovation networks. With the numerical simulation method, Choi et al. [35] made a comparison of the effectiveness of the innovation networks and found that scalefree network owns the highest learning performance, which is followed by broad-scale and single-scale network. This provided evidence for the significant impact of constraints on network effectiveness, which is proposed by Amaral et al. [29]. However, the structure of real network may differ widely from simulated ones, for example, in real network, most vertices may own relatively low connections, while a small ratio owns relatively high connections. In comparison, most vertices in simulated networks mostly own the same connections. Simulation of the real-world network has been conducted [42-45]. As the structures of real and simulated networks are different, it is necessary to make another empirical study with the real data, so that the learning performance of real networks with different network classification could be studied. This paper contributes to existing literatures by analyzing the real empirical network data with the simulation method. We examine the effectiveness of the real innovation network within the firms with simulation method. This would allow us to exclude other nonnetwork effects and conduct an independent study about the effect of innovation networks. We use the patent coauthorship data in establishing the innovation network and analyze the differences of real network structure and learning performance of 5 selected Chinese companies that filed the largest number of patents in China.

Section 2 classifies the small-world networks. Section 3 presents the computation analysis. Section 4 discusses and Section 5 concludes.

\section{Network Modeling}

2.1. Small Worldliness. Recent studies mostly utilized two key attributes to measure small world effect: local clustering and global average path length [3]. Clustering coefficient is measured with the number of links with which all neighbors of an inventor are connecting each other, divided by the number of connections among those inventors. For inventor $i$, the clustering coefficient is

$$
\mathrm{CC}_{i}=\frac{\tau_{i, \Delta}}{\tau_{i, 3}},
$$

where $\tau_{i, \Delta}$ is the number of triangles that contains inventor $i$ and $\tau_{i, 3}$ is the number of connected triples for which the other two inventors are both connected with $i$. The clustering coefficient (CC) of the network is averaged over all the inventors in the network.
The average path length (PL) is defined to be the average of all the shortest distances between any two inventors. For inventor $i$, the average path length is

$$
\mathrm{PL}_{i}=\frac{1}{n-1} \sum_{j=1, j \neq i}^{n}\left(\mathrm{PL}_{i, j}+1\right)
$$

where $\mathrm{PL}_{i, j}$ is the number of intermediaries between inventor $i$ and $j$. If $i$ and $j$ are directly connected, $\mathrm{PL}_{i, j}=0$. The average path length (PL) of the network is averaged over all the inventors in the network.

The small world network is characterized by high local clustering and low distance between inventors, which is distinguished from both random networks that are characterized by low clustering and low average path length and regular networks that are characterized by high clustering and high average path length. However, considering only the real network would be misleading, since the clustering coefficient may be overestimated and the patent length may be underestimated [46]. Following Newman et al. [46] and Humphries and Gurney [47], the CC ratio (CC of the real network/CC of a random network comparison) and $\mathrm{PL}$ ratio (PL of the real network/PL of a random network comparison) is introduced so that the estimation could be corrected. The more its CC ratio is greater than 1 and the less its PL ratio, the stronger the small world character is. It is usually measured by the small world quotient (CC ratio/PL ratio) $[5,7,26]$. The larger the quotient is, the greater the network's small world nature is. Humphries et al. [47] and Humphries and Gurney [48] proposed a definition that makes a judgment of the small world network: if $\mathrm{PL}$ ratio $\geq 1$ and $\mathrm{CC}$ ratio $\gg 1$, in other words, CC ratio/PL ratio $>1$, the network is said to be a small world network. Since PL is based on a connected component, both CC and PL are calculated within the largest connected component in this study.

All the indicators, such as CC, PL, and small world quotient, are calculated on the basis of unweighted network. The CC and PL of random network, with both the same number of patents and inventors and equal possibility that two inventors collaborate, are calculated with the method presented by Li et al. [45].

We identify the collaboration of inventors by utilizing the patent coauthorship data; that is, a link is established between two identified inventors as they coinvent a patent. We utilize the patent coauthorship data from SIPO (State Intellectual Property Office of China), which covers a total of over 6 million patents by the year 2011. The number of patents filed during 1992-2010 in SIPO grows at the fastest pace and becomes the second largest database in the world, only less than USPTO (United States Patent and Trademark Office) but greater than JPO (Japanese Patent Office) and EPO (European Patent Office) [18]. The patents in SIPO are classified into 3 categories: invention patent, utility model, and design. Since advanced technologies in China are mostly filed as invention patents [33], we utilize the data of invention patents filed by 5 Chinese companies with the largest number of patent application: two most famous MNCs in IT industry: Huawei and ZTE (Zhongxing Telecommunications Equipment), two largest state-owned MNCs in petroleum industry: 
TABLE 1: Summary statistics of patent coauthorship data in the largest connected component.

\begin{tabular}{lccccc}
\hline & $\begin{array}{c}\text { Size of largest } \\
\text { component }^{\mathrm{a}}\end{array}$ & $\begin{array}{c}\text { M: number of } \\
\text { patents }\end{array}$ & $\begin{array}{c}N \text { : number of } \\
\text { inventors }\end{array}$ & $\begin{array}{c}U: \text { average number of } \\
\text { patents an inventor filed }\end{array}$ & $\begin{array}{c}V: \text { average number of } \\
\text { inventors in a patent }\end{array}$ \\
\hline Foxconn & 0.5117 & 6,019 & 1,839 & 7.1147 & 2.1738 \\
Huawei & 0.9064 & 15,690 & 9,059 & 4.2328 & 2.4439 \\
SINOPEC & 0.8439 & 5,210 & 4,879 & 5.5433 & 5.1912 \\
CNPC & 0.8061 & 1,302 & 3,853 & 2.4269 & 7.1820 \\
ZTE & 0.9174 & 11,878 & 7,050 & 4.1417 & 2.4582 \\
\hline
\end{tabular}

${ }^{a}$ size of largest component is calculated with the ratio of inventors in the largest connected component to the total inventors.

SINOPEC (China Petroleum \& Chemical Corporation) and CNPC (China National Petroleum Corporation), and the large manufacturer Foxconn in China are included.

Since we analyze the innovation network within the company only, we remove the patents coapplied by two or more organizations (mainly composed of companies, universities, and research institutes) from the data, so that the interorganizational network is not taken into account. However, as there are always subsidiaries for large companies, patents coapplied by the parent firm and its subsidiary are included.

People usually own the same name in China, which may mislead the analysis of network structure. It is thus quite necessary but time consuming to distinguish inventors with identical name. However, as we have classified the data by company, it is scarce to see this phenomenon within a company.

The patent data in this study are filed during 20002009. Since we study only the largest connected network, we present the summary statistics of only the largest connected component of each company. As is shown in Table 1, the manufacturer Foxconn owns the smallest size numbered at 0.5117 , which suggests that the innovation network in Foxconn may not be well connected as there are $48.83 \%$ isolated inventors. In comparison, ratio of isolated inventors in the network of IT giants Huawei and ZTE is much lower, which are both lower than $10 \%$. Similarly, the size of largest component of petroleum giants SINOPEC and CNPC is also close.

Although Foxconn owns the fewest inventors, it owns more patents than SINOPEC and CNPC, which suggests that inventors in Foxconn may have done more intensive research and thus filed averagely more patents than the other four companies. In addition, as is shown in the last column, there are on average more inventors cofiling a patent in SINOPEC and CNPC than the others. This may either be because $R \& D$ project in petroleum industry requires more cooperative work or because of the bureaucracy of stateowned companies that respect managers by adding their name to the patent, even if they do not contribute to the patent.

In summary, the statistics of largest connected component differs by company. However, they show a similar trend by industry, which suggests that similar industrial invention may lead to common ground of innovation network.
TABLE 2: Small worldliness of innovation network.

\begin{tabular}{lccc}
\hline & Clustering coefficient & Path length & Small world Q \\
\hline Foxconn & 0.6775 & 10.4484 & 1.4443 \\
Huawei & 0.6213 & 5.4428 & 2.3294 \\
SINOPEC & 0.7764 & 5.3747 & 2.3893 \\
CNPC & 0.8223 & 6.0095 & 1.3545 \\
ZTE & 0.5544 & 5.7833 & 1.8819 \\
\hline
\end{tabular}

As is shown in Table 2, the small world Qs of these 5 MNCs are all greater than 1, which confirms the existence of small world characteristics. CNPC owns a higher clustering coefficient than the other companies, which may facilitate knowledge flow. In comparison, a longer path length between inventors in Foxconn may impede knowledge flow.

2.2. Classification of Network Structure. Differing clustering coefficient, path length, and small world Q suggest different network structures and thus learning performance of $5 \mathrm{com}$ panies. We may further investigate this from the perspective of distribution of connections. Since the characteristics of the tail of distribution determine the main effect on learning performance $[29,35]$, we conduct an in-depth analysis on it. As is shown in Figure 3, the tail of $\ln$-ln cumulative distribution curve of SINOPEC falls on a straight line, indicating an exponential decay of the distribution of connectivities and a broad-scale network according to Amaral et al. [29]. Comparing SINOPEC with an exponential decay, the tails of Huawei and ZTE appear to be falling faster, suggesting a Gaussian decay and broad-scale or single-scale networks. Since both exponential and Gaussian decays indicate that the connectivity distribution is not scale-free [29], we may conclude that there may be inefficiencies in knowledge flow in the real network according to Choi et al. [35].

The cumulative distribution curves of Foxconn and CNPC appear to be complex, with a convex in the tail that dislikes any distribution curve of classified networks mentioned above. Such weird distribution suggests a network with new structure. As is shown in Figure 4, for Foxconn, CNPC, and SINOPEC, there is a flat area in the tail of density curve, which indicates extremely unevenly distributed networks that own minor "super-inventors" who have extremely high connections with other inventors, while for most inventors in the network, the connections are much 

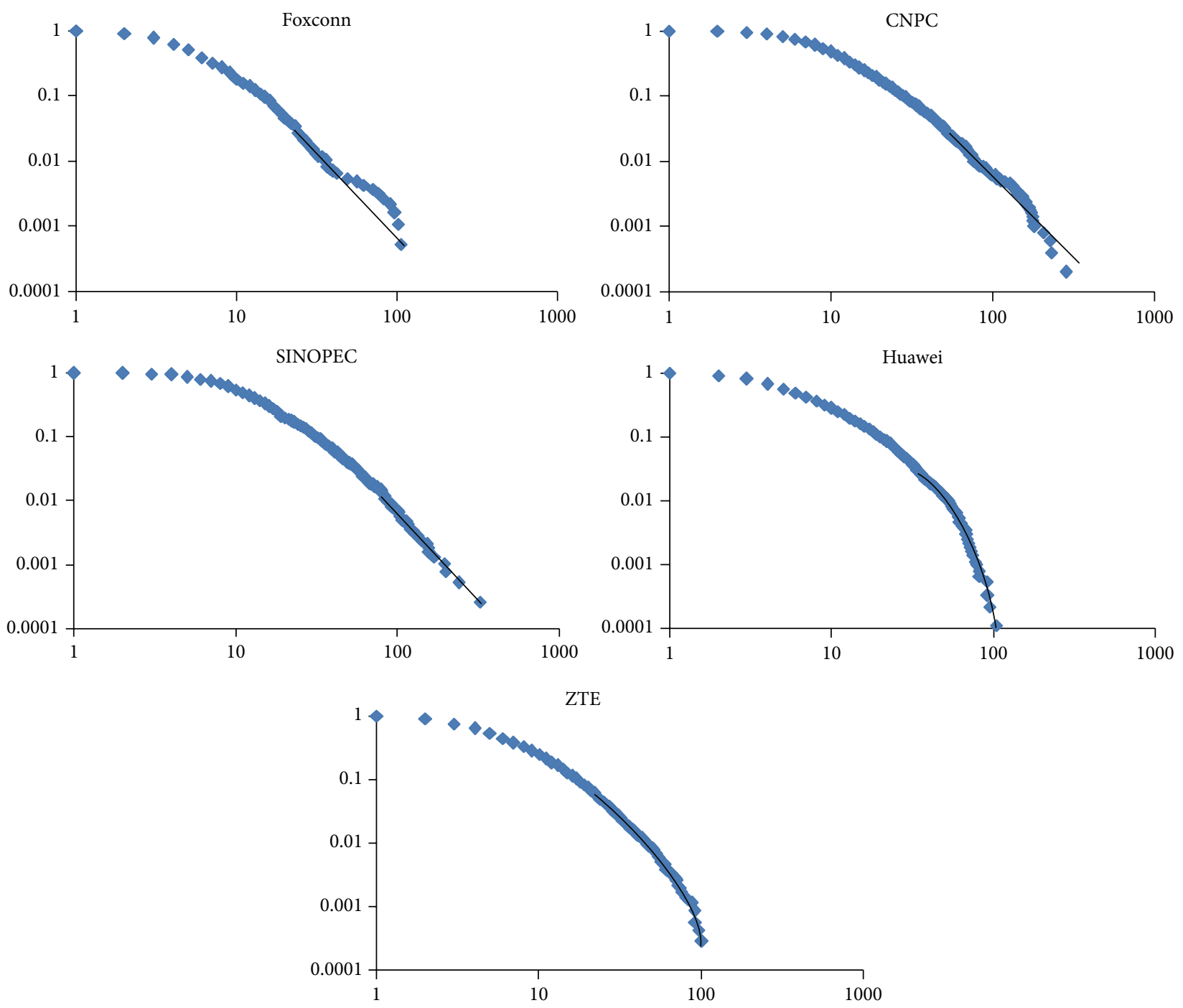

Figure 3: $\ln$-ln cumulative distribution function of connections. $x$-axis: $\ln$ (value) of inventors' degree as measured by the number of connections to other inventors; $y$-axis: $\ln$ (value) of cumulative distribution.

fewer. These lead to networks with hierarchical properties. In comparison, this phenomenon is absent from Huawei and ZTE with a smooth density curve, which indicates more evenly distributed networks where there appears to be no "super-inventors."

As is circled in Figure 4, the scale of the circled flat tails is different. Foxconn owns larger flat tail (0.0049) than CNPC (0.0041), which in turn is larger than SINOPEC (0.0036). A relatively larger flat tail appears to have produced a new structured network, while a relatively smaller flat tail is bound to be scale-free.

Therefore, there is monopolization in terms of technology innovation, which suggests that minor inventors may have controlled most R\&D resources and made most inventions by connecting with more other inventors. This monopolization appears to be greater in Foxconn than in CNPC and SINOPEC, while there appears to be no such monopolization in Huawei or ZTE.
2.3. How Important Is Innovation. For firms in different industry, the importance of innovation may be different. As these 5 companies are in different industries, they may make different effort in R\&D. Of all these 5 companies, Huawei and ZTE are likely to do more R\&D work, since information technology is typically an R\&D intensive industry [49]. In petrochemical technology industry in China, SINOPEC and CNPC are also devoted to build up many research institutes and research centers in technology fields of petrochemicals, coal-to-chemicals, commodity polymers, oilfield chemicals, and specialty chemicals (SINOPEC: http://english.sinopec.com/about_sinopec/subsidiaries/research_institutions/ 20080326/3092.shtml CNPC: http://www.cnpc.com.cn/en/ aboutcnpc/technologyinnovationRandDProgress/). In comparison, Foxconn, the largest gadget manufacturer in China, is doing more package work for R\&D intensive products, for example, iPhone and iPad.

Companies with a greater focus on innovation are more likely to be motivated to improve network efficiency. With 

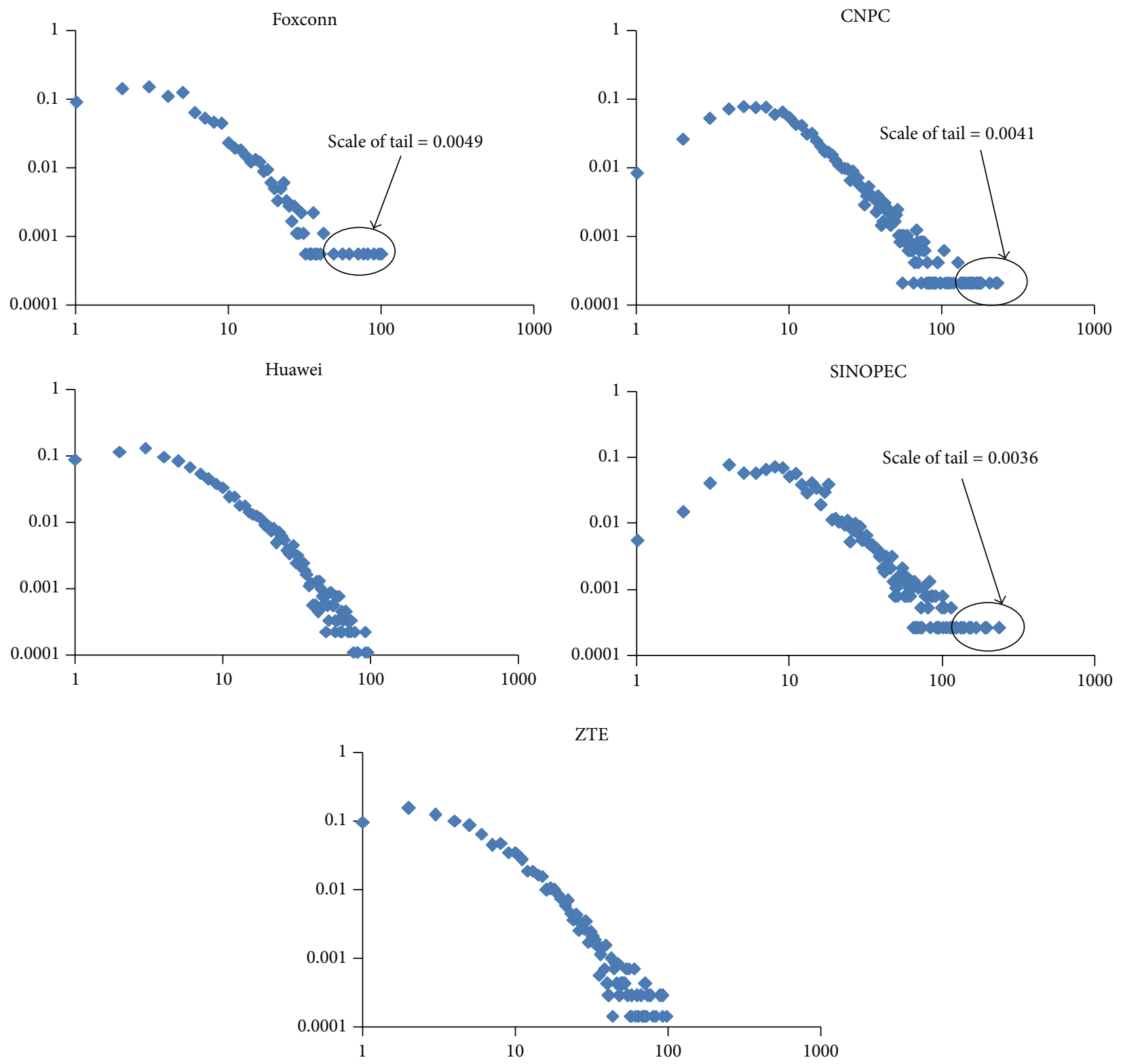

FIGURE 4: $\ln$-ln density curve of connections (note). Scale of tails is measured with the ratio of circled inventors.

the intention of identifying the importance of innovation by company, we use the data of industrial output and new product output for analysis. Since the value of high tech product is reflected by the market acceptance [50], selected indicators can also reflect the learning performance from the perspective of market value. As is shown in Table 3, although the scale of Foxconn, Huawei, and ZTE is in the same level in terms of industrial output, Foxconn produced much less new product than Huawei and ZTE. In comparison, for the sake of national monopolization, SINOPEC and CNPC own significantly higher industrial output; however, the new product output appears to be in the same level as Huawei and ZTE. In terms of the ratio of new product output, Huawei and ZTE perform better with higher ratio than SINOPEC and CNPC, while Foxconn owns the lowest performance with lower ratio than the others. As Foxconn do more assembly line production, technology innovation appears to be less important, which makes it less likely to pay much attention to improving the efficiency of innovation network.

Since the ratio of new product output differs by industry and by firms, it is reasonable to conclude that innovation in assembly line manufacturing may thus not be as important as in IT and petroleum industry, which may lead to certain network structures with different effects on learning. Companies that make more effort in R\&D work may pay more attention to network design and make more changes that will greatly improve network's performance; thus efficient innovation networks are more likely to be established. On the contrary, manufacturing oriented companies may provide production environment or even set the barriers that impede 
TABLE 3: Industrial output and new product output of 5 companies (billion RMB in 2010 price).

\begin{tabular}{lcccc}
\hline & Industrial output & New product output & Ratio of new product output & Year of data included \\
\hline Foxconn & 604.01 & 3.27 & 0.0054 & $2002,2007,2009,2010^{\mathrm{a}}$ \\
Huawei & 466.87 & 88.64 & 0.1899 & $2000,2002,2003,2005-2007,2009,2010$ \\
SINOPEC & $5,147.08$ & 189.01 & 0.0367 & $2000,2002,2003,2005-2007,2009,2010$ \\
CNPC & $4,112.46$ & 36.58 & 0.0089 & $2000,2002,2003,2005-2007,2009,2010$ \\
ZTE & 164.72 & 132.13 & 0.8021 & $2002,2003,2006,2007,2009,2010$ \\
\hline
\end{tabular}

Data resources: China Industry Business Performance Data (2001-2011).

${ }^{a}$ As the new product output for each company is not recorded all the year, we select the year of data where new product output is recorded.

innovation, so that the rule of standard product manufacturing is followed; thus the structure of innovation network is not likely to be improved.

\section{Computation Analysis}

With the intention of distinguishing the effectiveness of networks with different structure, we compare firm learning performance by investigating the efficiency of knowledge flow. Let the innovation network be formulated by innovative individuals (denoted by vertex) and their coauthorships (denoted by edge). We use the method of numerical simulation in measuring the effectiveness of knowledge flow based on the real network of 5 companies. In the initial period, we set individuals own heterogeneous knowledge. As the interactions increase, individuals will make a comparison between their own and neighbors' performance. When the individual has lower performance than one of his/her neighbors, he/she is more likely to learn from individuals with higher performance and update his/her own knowledge.

Accordingly, we refer to and extend the organization learning model by March [34]. First, we regard reality as having $m$ dimensions. Since the scale of network to be investigated is relatively large, we extend the sampling interval of each dimension $a_{s}(s=1,2, \ldots, m)$ by making $a_{s}$ randomly drawn from the integer set $[-13,-12,-11,-10,-9,-8,-7$, $-6,-5]$ and $[5,6,7,8,9,10,11,12,13]$ with equal probability. Second, we assume there are $n$ innovative individuals that are mutually interactive. The heterogeneous knowledge about the reality is also denoted by a vector with $m$ dimensions, $X_{j, 0}=\left[x_{1,0}, x_{2,0}, \ldots x_{m, 0}\right]$; each dimension will have a value randomly drawn from integer interval $[-13,13]$ (other intervals do not change our empirical result) with equal probability. The sampling interval of individuals is obviously larger than the reality by adding $[-4,-3,-2,-1,0,1,2,3$, 4], which suggests that individual owns no knowledge about the reality in this dimension (March (1991) sets $a_{s}$ to be randomly drawn from $[-1,1]$ with equal probability and $x_{s}$ be randomly drawn from $[-1,0,1]$. 0 suggests individual owns no knowledge about the reality in dimension $s$. Obviously we extend the organization learning model by March (1991) in this study). Since the reality is unknown to any individuals, the knowledge level $k_{j}$ of individual $j$ is determined by the distance between his/her heterogeneous knowledge and the reality; that is,

$$
k_{j}=\frac{1}{\sum_{s=1}^{m}\left(x_{s, j}-a_{s}\right)^{2}} .
$$

$\left(x_{s, j}-a_{s}\right)^{2}$ denotes the distance between $j$ 's heterogeneous knowledge and the reality in dimension $s$. Obviously, the closer the distance is, the smaller $\left(x_{s, j}-a_{s}\right)^{2}$ will be and the higher $k_{j}$ will be. Therefore, a high value of $k_{j}$ suggests a high level of knowledge.

The knowledge level determines the learning performance to a large extent [34]. However, since the result of innovation activity is highly mobile, it is appropriate to assume that learning performance is a variable and has its own distribution function. March [34] assumed learning performance was normally distributed and argued that higher knowledge level will increase both the expected performance and its variability. March [34] gave an explanation from the perspective of relative competition advantage: if there are $\mathrm{N}+$ $1(N>1)$ innovative individuals, the probability that the $N+$ 1 th individual performs better than the other $N$ individuals is usually determined by the area of right hand distribution (which is determined by the variability of performance) of the performance of $N+1$ th individual. A similar argument can also be found in David [51]. Improving the knowledge level will on the one hand increase the expected performance, but on the other hand will also increase the variability [34]. For example, adoption of a new technology will increase the output theoretically; however, since workers are not familiar with the new technology, the output is more likely to fluctuate in the short run; introducing an individual with heterogeneous knowledge, culture, and attitude into the organization will have a similar effect. As is stated above, it is reasonable to assume that the learning performance $y_{j}$ of individual $j$ is normally distributed with both expectation and variance being $k_{j}$; that is,

$$
y_{j} \sim N\left(k_{j}, k_{j}^{2}\right) .
$$

In the innovation network, the result of mutually learning process is to a large extent determined by the structural characteristics and network openness [35]. Let individual's openness be denoted by learning rate $p$, namely, the probability that individual $j$ changes his/her knowledge to $i$, who owns the highest performance of all $j$ 's partner. We set all the knowledge dimension change independently; then individual $j$ changes his $s$ th dimension knowledge $x_{s, j}$ to $i$ 's $x_{s, i}$ with probability $p$. A high value of $p$ suggests high openness, which suggests that individual $j$ is more likely to accept knowledge from outside. Choi et al. [35] and March [34] assign the value for $p$ in the interval $[0,0.9]$. Accordingly, we set $p$ to be $0.1,0.3$, and 0.9 in accordance with the network 


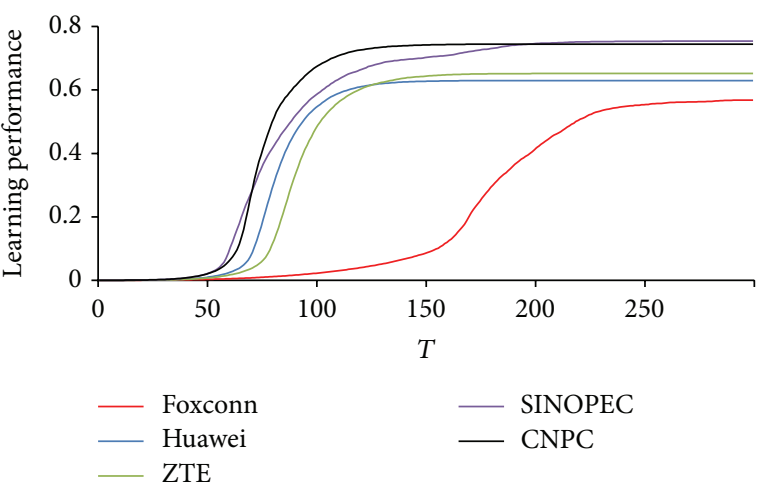

(a) $p=0.1$

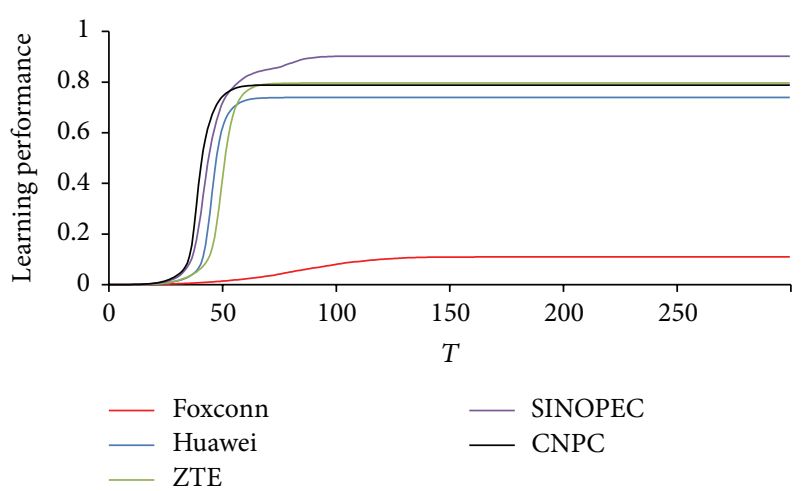

(b) $p=0.3$

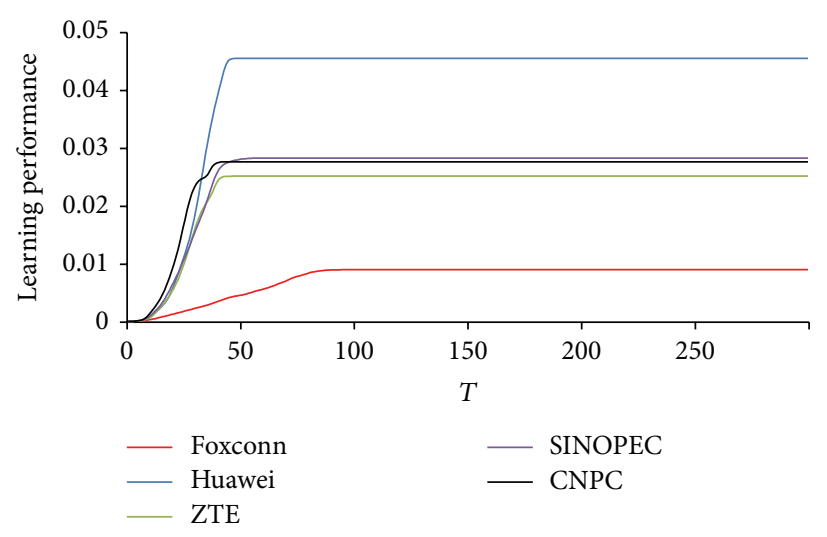

(c) $p=0.9$

FIGURE 5: Learning performance of network with different openness.

with low, middle, and high openness. We measure the network learning performance $\bar{y}$ by averaging the performance of all individuals in the network; that is, $\bar{y}=\sum_{i=1}^{N} y_{i}$. All the empirical results are averaged over 1,000 simulations.

As is shown in Figure 5, the learning performance remains flat in the initial period then rises sharply and converges to a constant value. This trend is similar with Choi et al. [35], whose findings are based on simulated network. Learning performance of networks with lower openness $\left(p_{0}=0.1\right)$ converges at lower rate but to a higher value; on the contrary, learning performance of networks with higher openness $\left(p_{0}=0.9\right)$ converges at higher rate but to a much lower value. It appears that network openness contributes positively to convergence rate but negatively to convergence value. This also corresponds with Choi et al. [35], who believe that substantially high openness homogenizes the entire cluster dramatically fast, coming to an equilibrium point before new knowledge is learned that could improve performance, resulting in lower performance throughout the cluster. In comparison, low openness facilitates individuals in achieving higher performance.

Innovation network of Foxconn has the lowest performance with a much lower convergence value than the other 4 networks in all periods. SINOPEC and CNPC own higher learning performance with low network openness, while Huawei owns the highest learning performance with high network openness in later period.
However, the scale of innovation networks of 5 companies is not equal, which may lead to the efficiency differences of knowledge flow between networks. A direct comparison of learning performance should be based on networks with the same scale. Ignoring the network scale may be misleading, for example, learning performance of network with 2 vertices would converge faster at a lower value than network with 10 vertices. However, we may propose that if the real network facilitates knowledge flow much better, it should perform better than other networks with the same scale. Following Davis et al. [7], Newman et al. [46], and Uzzi et al. [5], who estimated the small world quotient of real network by taking into account the random network with the same scale, that is, the same number of edges and vertex, we choose the random network as the reference. We compare the learning performance ratio of real networks, which divides the value of learning performance of real network by the learning performance of random network with the same scale in each period.

As is shown in Figure 6, the converging value of most real networks (except Foxconn) is close to 1 in the later period in Figures 6(a) and 6(b), which suggests that the learning performance of real and random network with low and medium openness is finally almost equal. However, if the openness is high, the converging value of real network is much lower than the random network (see Figure 6(c)). This is because low openness allows individuals to learn from each other for longer periods by delaying the occurrence of 


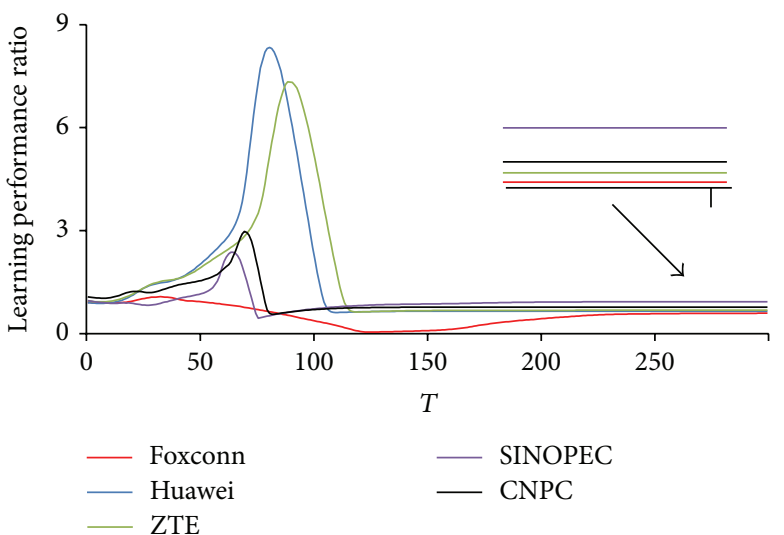

(a) $p=0.1$

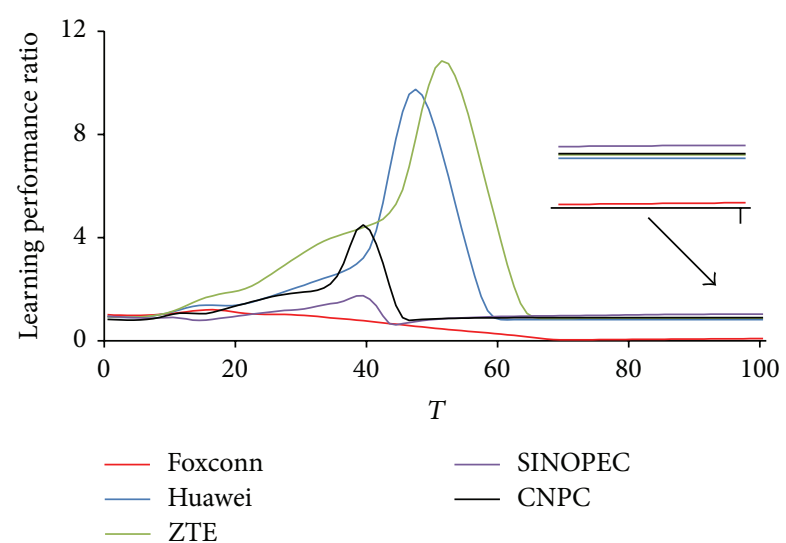

(b) $p=0.3$

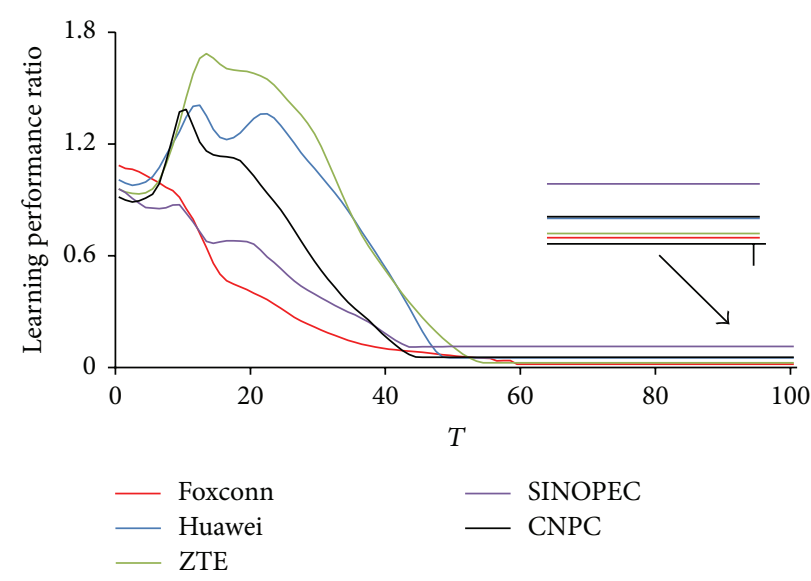

(c) $p=0.9$

FIGURE 6: Learning performance of network relative to random network.

convergence that terminates knowledge flow. On the contrary, knowledge flow in networks with high openness would stop earlier. In addition, the highest learning performance ratio in preconvergence period in (c) with high openness is also lower than in (a) and (b). As the individuals are closer to each other in random network, knowledge flow would be faster than in real network. Thus earlier convergence would facilitate individuals in random network.

The learning performance ratios of almost all the networks show inverted $U$ shape, which suggests that the learning performance of real network is initially becoming increasingly greater than the random network, but later the differences are becoming smaller.

After being divided by the learning performance of random network, the differences of converging value become smaller. However, the differences are also significant after zooming in the tail of the figure. The network of SINOPEC shows significantly higher convergence value than the other companies in Figures 6(a) and 6(c), while in (b) the differences are smaller. CNPC follows SINOPEC in the innovation convergence period. IT giants Huawei and ZTE are in the middle position. Foxconn owns the lowest converging value in all the network openness.
In initial period, IT giants Huawei and ZTE show much greater learning performance than the other three companies, which suggests that the network structure of Huawei and ZTE benefit knowledge flow better than the other networks in early period. However, they may not converge to higher value compared to the network of SINOPEC and CNPC.

In comparison, learning performance of Foxconn shows the lowest value in all periods, which suggests that the network structure of Foxconn owns the lowest efficiency of knowledge flow compared with the other 4 companies.

\section{Discussions}

The structure of innovation networks differs widely in reality, which may be the main cause of the differences of learning performance. Huawei and ZTE appear to facilitate learning better than Foxconn, CNPC, and SINOPEC in early period when performance has been increasing (see Figures 5 and 6), while the latter two companies converge to higher learning performance. As the largest manufacturer in China, learning performance in Foxconn appears to be the lowest, which may harm technology innovation according to Shin and Park [52] and Orihata and Watanabe [53]. With a careful analysis, 
we may find that the network structure of Foxconn is quite different from the other 4 companies, and also SINOPEC and CNPC are different from Huawei and ZTE, which can be summarized into the following 5 aspects that may be the main cause of learning performance differences.

1st: the innovation network of Foxconn is not well connected, with a smaller size of largest connected component than the other four companies (see Table 1). This would obviously cut the path of knowledge flow and prevent knowledge exchanges between inventors. As inventors are more distant from each other in Foxconn than in the other 4 companies (see Table 2), knowledge flow will have to pass more intermediaries and thus lose efficiency.

2nd: there is network monopolization in Foxconn, CNPC, and SINOPEC, with relatively small ratio of "super-inventors" inventing most technologies (see Figure 4). Since most inventors are connected with these "super-inventors," innovation networks in the above 3 companies are likely to be hierarchical, where minor inventors account for large ratio of $R \& D$ resources by acting as the most important intermediaries of knowledge flow. This network structure may prevent efficient knowledge flow, since inventors with heterogeneous knowledge are not likely to take important positions. People are more likely to learn from "super-inventors" which leads to knowledge homogeneity that harms innovation. This may be another cause of a lower learning performance of Foxconn (see Figures 5 and 6), whose network monopolization is greater than in CNPC and SINOPEC (see Figure 4).

3rd: the network monopolization in SINOPEC leads to a network structure that to some degree harms innovation. As is shown in Figure 6, SINOPEC owns higher performance than Huawei and ZTE in later period, which is mainly due to a scale-free structured network that owns fewer constraints than broad-scale or single-scale networks [29]. However, under the environment of hierarchy characterized by staterun companies, "super-inventor" monopolized networks may harm innovation. This may be the main cause that the learning performance of SINOPEC with scale-free networks falls far behind Huawei and ZTE with broad-scale or singlescale networks in early period, which is different from Choi et al. [35], who found that scale-free network performs better than broad-scale and single-scale networks in all the periods.

4th: the two state-run petroleum giants, SINOPEC and CNPC, have different network structure. The tail of SINOPEC is likely to be scale-free (see Figure 3), while CNPC is not. This may be mainly because CNPC owns higher monopolization level than SINOPEC, which may be the main cause that SINOPEC appears to own more efficient knowledge flow in later period than CNPC (see Figures 5 and 6). However, as a state-run company characterized by bureaucracy, neither SINOPEC nor CNPC could avoid establishing a hierarchical network that suffers efficiency losses.

5th: a relatively flat network structure like Huawei and ZTE (see Figure 4) with R\&D resources evenly distributed may facilitate learning. However, as the network appears to be broad-scale or even single-scale, there are still efficiency losses, which make Huawei and ZTE perform worse than SINOPEC and CNPC in later period.
The causes that lead to the differences of network structure may be, on the one hand, the attention paid to the innovation network. As the technology innovation in Foxconn is not so important (see Table 3), it may pay much less attention to improving the network structure and will allow the existence of low efficient innovation network and, on the other hand, the genetic bureaucracy of state-run firms. Although CNPC and SINOPEC pay much attention to innovation, bureaucracy leads to a hierarchical network that is losing efficiency. In addition, since Huawei and ZTE have paid much attention to innovation and are free from bureaucracy, they established relatively efficient innovation networks that facilitate knowledge flow better when learning performance is increasing.

\section{Conclusions and Limitations}

This paper uses the patent coauthorship data of China in establishing the innovation network of 5 MNCs that filed the largest number of patents in China. The structure of network differs by company and by industry: inventors in the largest Chinese manufacturer Foxconn are more distant from each other, which reduces the network efficiency; the network of petroleum giant SINOPEC is scale-free but connections are hierarchically distributed, while IT giants Huawei and ZTE are broad-scale or single-scale but connections are flatly distributed. We establish the innovation network with the patent coauthorship data by these 5 MNCs and make a classification of these networks according to the distribution curve of connections. We develop an organization learning model by regarding the reality as having $\mathrm{m}$ dimensions, which denotes the heterogeneous knowledge about the reality. We set $n$ mutually interactive innovative individuals, who own unique knowledge about the reality. The distance between the knowledge of the individual and the reality denotes the knowledge level of that individual. In the empirical analysis section, we make 1,000 numerical simulations by randomly assigning values to each dimension and get the learning performance of these 5 MNCs. We then make a comparison of the learning performance of these $5 \mathrm{MNCs}$ with differing network structures, where individuals interact with and learn from each other within the small world network. The empirical result shows that different network structures lead to differing learning performance: Huawei and ZTE perform better in early period, which is attributed to a flat distribution of connections that allows inventors with heterogeneous knowledge to take important positions. However, as the networks of Huawei and ZTE are broad-scale or single-scale, efficiency losses are significant in later period. The learning performance of Huawei and ZTE appears to be lower than SINOPEC with scale-free network in later period. In comparison, for Foxconn where technology innovation is less important, the learning performance is the lowest.

Our empirical finding is implicative for company managers from the following aspects. (1st) As carefully and well organized networks (e.g., Huawei, ZTE, CNPC, and SINOPEC with much focus on innovation) would show higher efficiency in knowledge flow and thus perform better 
than randomly organized networks, and with not well organized networks (e.g., Foxconn with less focus on innovation), it is quite necessary to make a careful reorganization of the innovation network, even for firms where innovation is less important. (2nd) Since network monopolization may be harmful to innovation, it is beneficial to transfer a hierarchical network to a flat network, where R\&D resources are evenly distributed among inventors. (3rd) Since perfectly scale-free networks may never exist in reality, making firm innovation network structure closer to scale-free may be more favorable.

As the learning performances of inventors within the network are simulated, how simulation results correspond with the real learning performance is not analyzed in this study. As the real learning performance is determined by multireal factors, it is necessary to conduct a survey with inventors about these potential impacting factors. Our future attention would possibly be paid to testing the consistency of the simulated learning performance with the survey data of these 5 firms.

\section{Conflict of Interests}

The authors declare that there is no conflict of interests regarding the publication of this paper.

\section{Acknowledgments}

This work is supported by the National Natural Science Foundation of China (NSFC: 71173009), Beijing Natural Science Foundation (9144036), and Ministry of Education, Humanities and Social Sciences Project (13YJC630219).

\section{References}

[1] M. Dodgson and R. Rothwell, Handbook of Industrial Innovation, Edward Elgar, Cheltenham, UK, 1994.

[2] S. Milgram, "The small world problem," Psychology Today, no. 1, pp. 60-67, 1967.

[3] D. J. Watts and S. H. Strogatz, "Collective dynamics of "smallworld” networks," Nature, vol. 393, no. 6684, pp. 440-442, 1998.

[4] T. Hellmann and M. Staudigl, "Evolution of social networks," European Journal of Operational Research, vol. 234, no. 3, pp. 583-596, 2014.

[5] B. Uzzi, L. A. Amaral, and F. Reed-Tsochas, "Small-world networks and management science research: a review," European Management Review, no. 4, pp. 77-91, 2007.

[6] L. Björneborn, "Mini small worlds' of shortest link paths crossing domain boundaries in an academic Web space," Scientometrics, vol. 68, no. 3, pp. 395-414, 2006.

[7] G. F. Davis, M. Yoo, and W. E. Baker, "The small world of the American corporate elite, 1982-2001," Strategic Organization, vol. 1, no. 3, pp. 301-326, 2003.

[8] J. H. Fowler, "Turnout in a small world," in Social Logic of Politics, A. Zuckerman, Ed., Temple University Press, Philadelphia, Pa, USA, 2005.

[9] B. Kogut and G. Walker, "The small world of Germany and the durability of national networks," American Sociological Review, vol. 66 , no. 3, pp. 317-335, 2001.
[10] J. Kleinberg, "Navigation in a small world-It is easier to find short chains between points in some networks than others," Nature, vol. 406, p. 845, 2000.

[11] S. M. Iravani, B. Kolfal, and M. P. Van Oyen, "Call-center labor cross-training: it's a small world after all," Management Science, vol. 53, no. 7, pp. 1102-1112, 2007.

[12] S. Goyal, M. J. van der Leij, and J. L. Moraga-González, "Economics: an emerging small world," Journal of Political Economy, vol. 114, no. 2, pp. 403-412, 2006.

[13] J. A. C. Baum, A. V. Shipilov, and T. J. Rowley, "Where do small worlds come from?" Industrial and Corporate Change, vol. 12, no. 4, pp. 697-725, 2003.

[14] D. J. Watts, Small Worlds: The Dynamics of Networks between Order and Randomness, Princeton University Press, Princeton, NJ, USA, 1999.

[15] L. C. Fleming and M. Marx, "Managing creativity in small worlds," California Management Review, vol. 48, no. 4, pp. $6-$ 27, 2006.

[16] A. Hargadon, How Breakthroughs Happen: The Surprising Trust About How Companies Innovate, Bridging Small Worlds, Harvard Business School Press, Boston, Mass, USA, 2003.

[17] Z. F. Chen and J. C. Guan, "The impact of small world on innovation: an empirical study of 16 countries," Journal of Informetrics, vol. 4, no. 1, pp. 97-106, 2010.

[18] J. C. Guan and Y. Shi, "Transnational citation, technological diversity and small world in global nanotechnology patenting," Scientometrics, vol. 93, no. 3, pp. 609-633, 2012.

[19] L. C. Fleming, C. King III, and A. I. Juda, "Small worlds and regional innovation," Organization Science, vol. 18, no. 6, pp. 938-954, 2007.

[20] S. W. Hung and A. P. Wang, "Examining the small world phenomenon in the patent citation network: a case study of the radio frequency identification (RFID) network," Scientometrics, vol. 82, no. 1, pp. 121-134, 2010.

[21] B. Verspagen and G. Duysters, "The small worlds of strategic technology alliances," Technovation, vol. 24, no. 7, pp. 563-571, 2004.

[22] P. P. Chen and S. W. Gao, "Observer-based feedback stabilization of networked control systems with random packet dropouts," Mathematical Problems in Engineering, vol. 2013, Article ID 218682, 7 pages, 2013.

[23] C. J. Carstens and K. J. Horadam, "Persistent homology of collaboration networks," Mathematical Problems in Engineering, vol. 2013, Article ID 815035, 7 pages, 2013.

[24] D. J. Watts, "Networks, dynamics, and the small-world phenomenon 1," American Journal of Sociology, vol. 105, no. 2, pp. 493-527, 1999.

[25] A. L. Barabási and R. Albert, "Emergence of scaling in random networks," Science, vol. 286, no. 5439, pp. 509-512, 1999.

[26] R. Albert, H. Jeong, and A. L. Barabási, "Diameter of the worldwide web," Nature, vol. 401, no. 6749, pp. 130-131, 1999.

[27] P. O. Seglen, “The skewness of science," Journal of the American Society for Information Science, vol. 43, pp. 628-638, 1992.

[28] S. Redner, "How popular is your paper? An empirical study of the citation distribution," European Physical Journal B, vol. 4, no. 2, pp. 131-134, 1998.

[29] L. A. N. Amaral, A. Scala, M. Barthélémy, and H. E. Stanley, "Classes of small-world networks," Proceedings of the National Academy of Sciences of the United States of America, vol. 97, no. 21, pp. 11149-11152, 2000. 
[30] D. J. Spielman, K. Davis, M. Negash, and G. Ayele, "Rural innovation systems and networks: findings from a study of Ethiopian smallholders," Agriculture and Human Values, vol. 28, no. 2, pp. 195-212, 2011.

[31] T. Van der Valk, M. M. H. Chappin, and G. W. Gijsbers, "Evaluating innovation networks in emerging technologies," Technological Forecasting and Social Change, vol. 78, no. 1, pp. 25-39, 2011.

[32] F. Hermans, D. Van Apeldoorn, M. Stuiver, and K. Kok, "Niches and networks: explaining network evolution through niche formation processes," Research Policy, vol. 42, no. 3, pp. 613-623, 2013.

[33] G. P. Zhang and J. C. Guan, "The impact of small world on patent productivity in China," Scientometrics, vol. 98, no. 2, pp. 945960, 2014.

[34] J. G. March, "Exploration and exploitation in organizational learning," Organization Science, no. 21, pp. 71-87, 1991.

[35] J. Choi, A. Sang-Hyun, and M. Cha, "The effects of network characteristics on performance of innovation clusters," Expert Systems with Applications, vol. 40, no. 11, pp. 4511-4518, 2013.

[36] B. T. Asheim and A. Isaksen, "Regional innovation systems: the integration of local "sticky" and global "ubiquitous" knowledge," Journal of Technology Transfer, vol. 27, no. 1, pp. 77-86, 2002.

[37] R. A. Boschma, "The rise of clusters of innovative industries in Belgium during the industrial epoch," Research Policy, vol. 28, no. 8, pp. 853-871, 1999.

[38] M. P. Feldman, J. Francis, and J. Bercovitz, "Creating a cluster while building a firm: entrepreneurs and the formation of industrial clusters," Regional Studies, vol. 39, no. 1, pp. 129-141, 2005.

[39] E. Giuliani and M. Bell, "The micro-determinants of meso-level learning and innovation: evidence from a Chilean wine cluster," Research Policy, vol. 34, no. 1, pp. 47-68, 2005.

[40] N. Gilbert, P. Ahrweiler, and A. Pyka, "Learning in innovation networks: some simulation experiments," Physica A: Statistical Mechanics and Its Applications, vol. 378, no. 1, pp. 100-109, 2007.

[41] R. Oakey, "Clustering and the R\&D management of hightechnology small firms: in theory and practice," $R$ and $D$ Management, vol. 37, no. 3, pp. 237-248, 2007.

[42] L. Zhang, W. Zhang, M. Xiao, J. Xie, and Z. Wu, "Unravelling gene networks from steady-state experimental perturbation data," in Proceedings of the 3rd International Conference on Bioinformatics and Biomedical Engineering (ICBBE '09), pp. 1-4, June 2009.

[43] L. Zhang, X. Ju, Y. Cheng, X. Guo, and T. Wen, "Identifying Tmem59 related gene regulatory network of mouse neural stem cell from a compendium of expression profiles," BMC Systems Biology, vol. 5, p. 152, 2011.

[44] L. Zhang, M. Xiao, Y. Wang, and W. Zhang, "Reverse engineering large-scale genetic networks: synthetic versus real data," Journal of Genetics, vol. 89, no. 1, pp. 73-80, 2010.

[45] Z. W. Li, X. H. Yang, F. L. Jiang, G. Chen, G. Q. Weng, and M. Zhu, "Dynamically weighted clique evolution model in clique networks," Mathematical Problems in Engineering, vol. 2013, Article ID 182638, 6 pages, 2013.

[46] M. Newman, S. H. Strogatz, and D. J. Watts, "Random graphs with arbitrary degree distributions and their applications," Physical Review E-Statistical, Nonlinear, and Soft Matter Physics, vol. 64, no. 2, Article ID 026118, pp. 261181-261187, 2001.

[47] M. D. Humphries and K. Gurney, "Network "small-worldness": a quantitative method for determining canonical network equivalence," PLoS ONE, vol. 3, no. 4, Article ID e2051, 2008.
[48] M. D. Humphries, K. Gurney, and T. J. Prescott, "The brainstem reticular formation is a small-world, not scale-free, network," Proceedings of Biological Sciences, vol. 273, no. 1585, pp. 503-511, 2006.

[49] Office of Technology Assessment United States Congress, Information Technology R\&D: Critical Trends and Issues Summary, U. S. Congress Office of Technology Assesment, New York, NY, USA, 1985.

[50] X. H. Liu and T. Buck, "Innovation performance and channels for international technology spillovers: evidence from Chinese high-tech industries," Research Policy, vol. 36, no. 3, pp. 355-366, 2007.

[51] H. A. David, Order Statistics, John Wiley, New York, NY, USA, 2nd edition, 1981.

[52] J. Shin and Y. Park, "Evolutionary optimization of a technological knowledge network," Technovation, vol. 30, no. 11-12, pp. 612626, 2010.

[53] M. Orihata and C. Watanabe, "Evolutional dynamics of product innovation: the case of consumer electronics," Technovation, vol. 20, no. 8, pp. 437-449, 2000. 


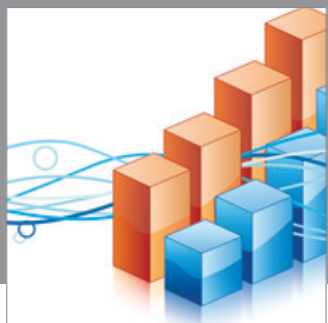

Advances in

Operations Research

mansans

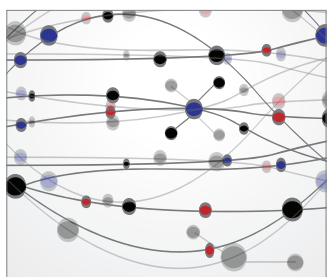

The Scientific World Journal
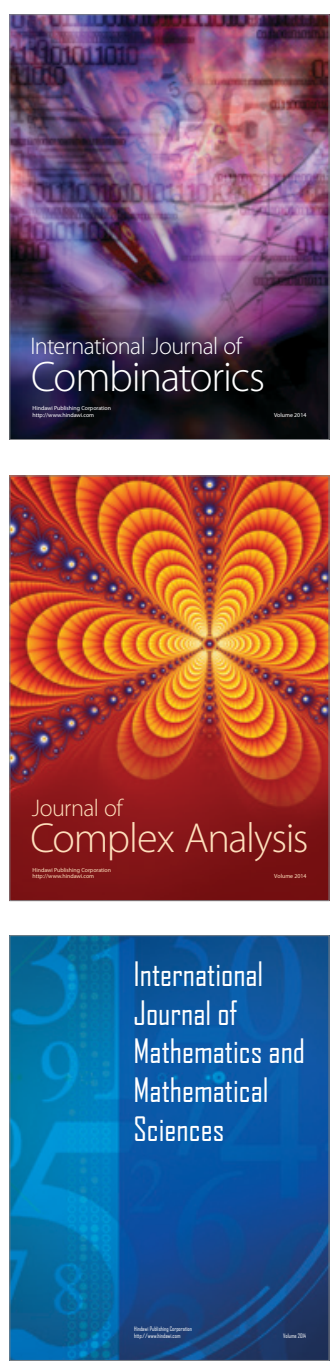
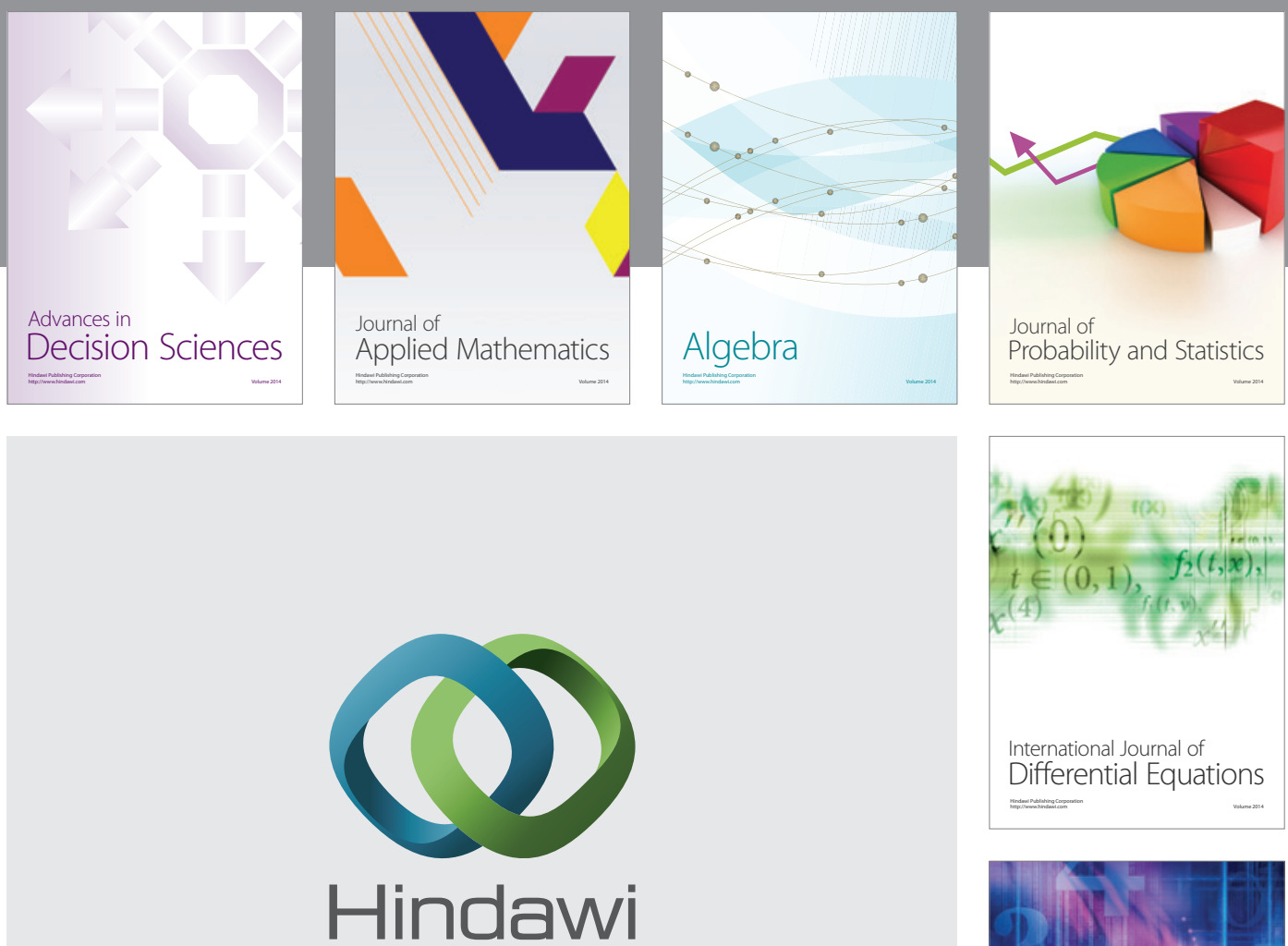

Submit your manuscripts at http://www.hindawi.com
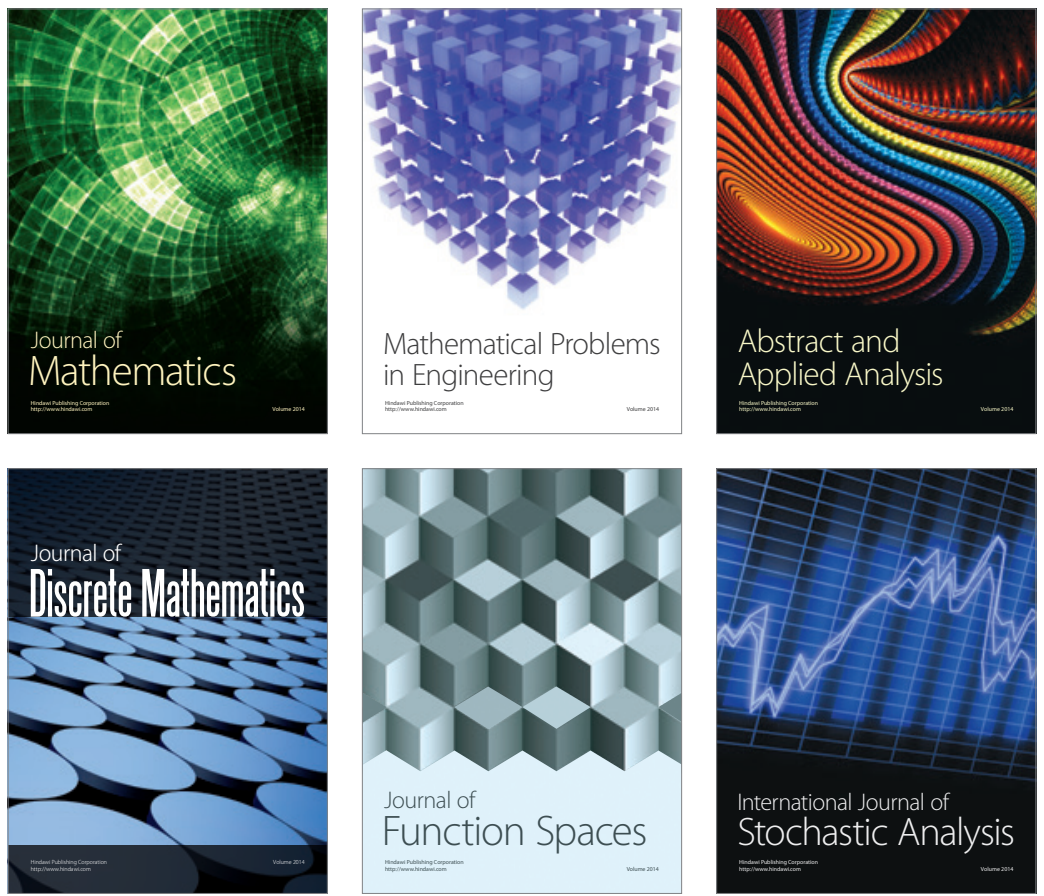

Journal of

Function Spaces

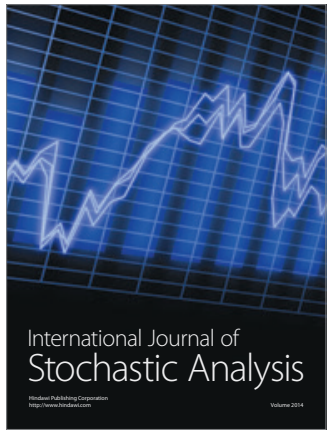

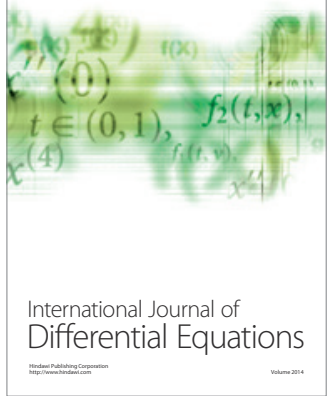
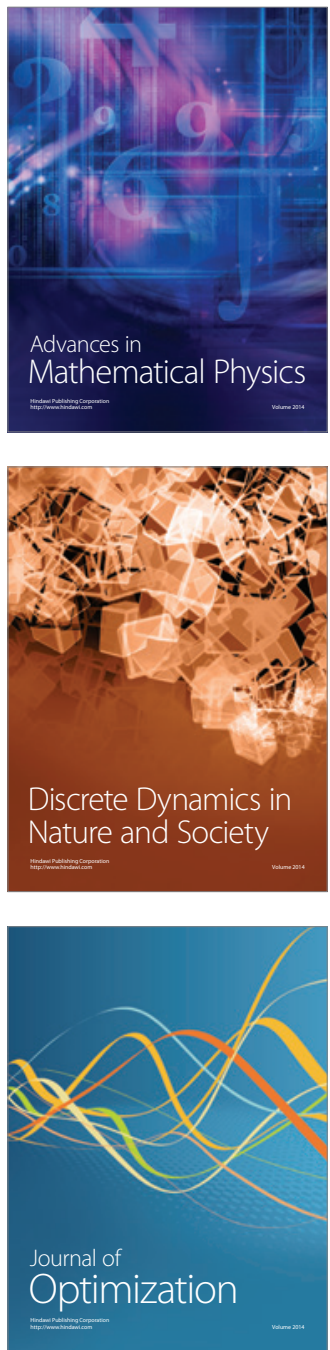